\title{
Study on Particle Swarm Algorithm to Solve the Problem of Shafting Rotating Error
}

\author{
Wu Yudong ${ }^{1}$ and Zhao Xuesen ${ }^{2}$ \\ ${ }^{1}$ School of Basic Science, Harbin University of Commerce, Harbin 150028, China \\ ${ }^{2}$ Center for Precision Engineering, Harbin Institute of Technology, \\ Harbin 150001, China \\ Wuyudong@aliyun.com
}

\begin{abstract}
For solving the rotation error problem of the shafting, a three-dimensional motion model was developed, and it turned into a multi-parameter optimization problem. As the multiparameter optimization problem solving approach, the particle swarm optimization (PSO) was introduced to calculate this problem, and carried out shaft rotary motion trajectory simulation experiments. The PSO algorithm to calculate the rotational error was prepared and developed an analysis software, the correctness of PSO algorithm was also verified. Finally, an example of rotational error measurement experiment by atomic force microscope (AFM) was calculated and obtained the rotational error results.
\end{abstract}

Keywords: Shafting; rotating error; multi-parameter optimization; PSO

\section{Introduction}

Rotary axis system is a critical component of processing engine bed and test equipment. With the enhancement of machining precision, requirements of the precision of rotary axis system are becoming higher and higher, so that many direct measuring methods could not meet the requirements of actual measurement. Thus, complex measuring adjustment process is required and turning errors are also decomposed into several components with different characteristics to be tested respectively, which, no matter in terms of efficiency or accuracy, is limited to certain degrees [1 3]. Generally speaking, precision main shaft turning errors could be approximately divided into two basic forms: axial end beat and radial turning error (including radial run-out and angle rotation. Nowadays, precision measuring methods of precision main shaft turning errors are mainly static measurement, dynamic multi-measuring heads and multiple bearing, etc. In a word, these measuring measures require either the most precise excircle standard profile as the standard or relatively complicated measuring equipment or complex measuring adjustment process; meanwhile the measuring goal aims also at the radial turning errors of the main shaft. If high accuracy sensor is adopted to have turning flash catch and analysis (i.e. shape matching analysis) of the shape and appearance of the sample ends which is vertical to the main axis, it is expected to realize the measurement of precision radial turning errors of the principle axis and the measurement of the error data of axial end beat at the same time, which would become a precision radial turning error measuring method with simple principle and wide application. From this point of view, this essay transforms solving turning errors into a calculating the multi-parameter optimization with three-dimensional shape data. No doubt, particle swarm optimization (PSO) is an effective way to solve the problem. To sum up, in terms of the measurement of turning errors 
of rotary axis system, this essay set up its mathematic model and introduces mainly how to solve this problem with PSO algorithm.

This paper is structured as follows: Section 2 introduce the basic principles and the Kinematic error model of rotary axis system; then, Section 3 describe the solving method: particle swarm optimization, and it's solving process; Section 4 develop a simulation software to calculate the shafting turning errors with PSO method, and verify its validity; Section 5 calculate a simple example of rotational error measurement experiment by Atomic force microscope(AFM) and obtain the rotational error results; Finally we discuss the conclusions in Section 6.

\section{Kinematic Error Model of Rotary Axis system}

Principle of measuring the kinematic errors of rotary axis system see reference $[4,5]$, which uses three-dimensional shape to obtain 3-D data by scanning, imaging and gauging the surface topography of axial turning flash position s. Measurement procedure is illustrated as shown in Figure 1(a): first, extract 3-D coordinate with various feature points from a 3-D data image, such as a feature point coordinate $\mathrm{P}_{1}\left(x_{1}, y_{1}, z_{1}\right)$; when the axis rotating to a known angle $\theta$, as shown in Figure 1(b), the feature point coordinate becomes $\mathrm{P}_{2}\left(x_{2}, y_{2}, z_{2}\right)$; if it is known that the rotation axis equation of the rotating process $L \mathrm{c}$, the coordinate $\mathrm{P}_{2}$ when $\mathrm{P}_{1}$ rotating $\theta$ surrounding the axis could be obtained. In reverse, if the coordinates of $\mathrm{P}_{1}$ and $\mathrm{P}_{2}$ as well as $\theta$ are already known, the equation of the axis of rotation $L c$ could be absolutely determined with various points (more than five). This is the variation of the axis of rotation we need, i.e. the kinematic error of the rotary axis system. By rotating continuously the uniformly-spaced angle $\theta$, the kinematic error curve of the rotary axis system on complete circle could be obtained.

Next the geometric transformation relation of this process would be deduced. Suppose the point equation of the axis is $\frac{x-x_{0}}{m}=\frac{y-y_{0}}{n}=\frac{z-z_{0}}{p}$, among which the straight line crossing point is $P_{0}\left(x_{0}, y_{0}, z_{0}\right)$ and the direction vector is $(m, n, p)$, and the coordinate schematic diagram is shown as in Figure 2(a).
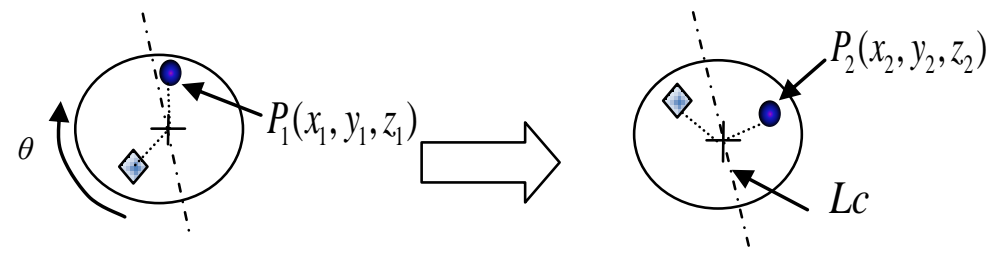
a) before rotating
b) after rotating

\section{Figure 1. Schematic diagram of the measurement principle}

First, suppose unit vector $u=(a, b, c)$ is the unit vector of straight line, as shown in Figure 2(a), then

$$
a=\frac{m}{\sqrt{m^{2}+n^{2}+p^{2}}}, \quad b=\frac{n}{\sqrt{m^{2}+n^{2}+p^{2}}}, \text { and } c=\frac{p}{\sqrt{m^{2}+n^{2}+p^{2}}} .
$$


Next we will start transforming the coordinate, as shown in Figure 2(b), in the sequence of which is:

1) translate the point $P_{0}\left(\mathrm{x}_{0}, \mathrm{y}_{0}, \mathrm{z}_{0}\right)$ to coordinate system origin and suppose its matrix as $\operatorname{Tr}$;

2) rotation operation: rotate this straight line till it overlaps with axle $\mathrm{Z}$, i.e., rotate angle $\alpha$ surrounding axle $\mathrm{X}$, whose matrix is $R_{x}(\alpha)$, and then rotate angle $\beta$ surrounding axle $\mathrm{Y}$ to axle $\mathrm{Z}$, whose matrix is $R_{y}(\beta)$;

3) rotate the feature point to the angle of $\theta$ surrounding $\mathrm{Z}$, whose rotation matrix is $R_{z}(\theta)$

4) reverse rotation $\beta$ surrounding axle $Y$, whose matrix is $R_{y}^{-1}(\beta)$, rotation $\alpha$ surrounding axle $\mathrm{X}$, whose matrix is $R_{x}^{-1}(\alpha)$,respectively;

5) reverse translate the origin to $P_{0}\left(\mathrm{x}_{0}, \mathrm{y}_{0}, \mathrm{z}_{0}\right)$, whose matrix is $\operatorname{Tr}^{-1}$.

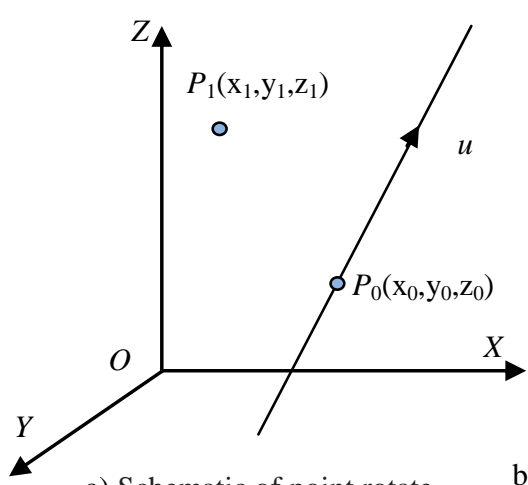

a) Schematic of point rotate with the line

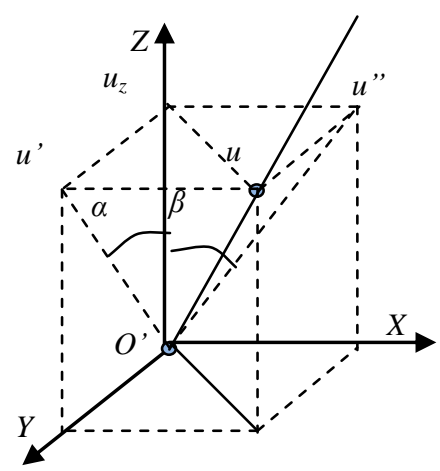

b) Translate $\mathrm{P}_{0}$ to $O$, rotate the line to $\mathrm{Z}$ axis along $\mathrm{X}$ axle and $\mathrm{Y}$ axle sequentially; and then perform these processes in reverse order

Figure 2. Coordinate transformation diagram

The matrix transformation relation of the above transformation is:

$$
R_{t o l}(\theta)=\operatorname{Tr}^{-1} \cdot R_{x}^{-1}(\alpha) \cdot R_{y}^{-1}(\beta) \cdot R_{z}(\theta) \cdot R_{y}(\beta) \cdot R_{x}(\alpha) \cdot \operatorname{Tr}
$$

Thus, the space coordinates transformation relation of these two points is:

$$
\left[x_{2}, y_{2}, z_{2}, 1\right]^{T}=R_{t o l}(\theta)\left[x_{1}, y_{1}, z_{1}, 1\right]^{T}
$$

Since the unknown parameters in the coordinate transformation matrix are more than 6 freedoms and are higher order terms, it is not easy to solve equation (2) with various data point simultaneous. Therefore, solving turning axis equation becomes a problem of the multi-parameter optimization, to which PSO calculation is an efficient way 


\section{Particle Swarm Optimization and the Solving Process}

Particle Swarm Optimization (PSO) put forward by Kennedy and Eberhart in 1995 is a global optimization based on swarm intelligence theory. It is to conduct optimizing search among swarm intelligences through the cooperation and changes among the particles in swarms. It is especially suitable for solving multi-parameter optimization, such as dynamic target optimization and multi-dimension optimization, with the advantages of fast rate of convergence and robustness [6 10]. At present, scholars have improved and obtained many practical variant of PSO calculation, among which the most practical is to introduce inertia factor to algorithm; thus, the update formula of the algorithm is as follows:

$$
\begin{gathered}
V_{i d}(t+1)=w_{i t e r} \cdot V_{i d}(t)+c_{1} r_{1} \cdot\left(P_{i d}(t)-X_{i d}(t)\right)+c_{2} r_{2} \cdot\left(P_{g t}(t)-X_{i d}(t)\right) \\
X_{i d}(t+1)=X_{i d}(t)+V_{i d}(t+1)
\end{gathered}
$$

The subscript $d$ in the formula refers to the dimension of the searching space, $\mathrm{X}_{i d}$ refers to current position of the $i$ particle, $\mathrm{V}_{i d}$ refers to current running velocity of the $i$ particulate, $P_{i d}$ refers to the best position the $i$ particulate has experienced, which is named as the best individual position; $P_{g t}$ (also called Gbest) is the best position that all the particulates have experienced. $c_{1}$ and $c_{2}$ are the coefficients of self-cognition and social cognition, which are used to adjust the step length of the best position direction and the global best position direction that the particles fly to. Generally, $c_{l}=c_{2}=2 ; r_{l}$ and $r_{2}$ are the random numbers averagely distributed. $w_{\text {iter }}$ is called inertia factor and generally $w_{\text {iter }}=w_{\max }-i$ ter $*\left(w_{\max }-w_{\min }\right)$ iter $r_{\max }$, among which iter is current iterations and iter $_{\max }$ is the total of the iterations.

The general solving process of PSO calculation is:

1) Initialize the parameters of the particle swarm, including the number of the particle swarms, random initial position and velocity, maximum iteration, etc.;

2) Calculate the adaptive value of every particle (i.e., the target function value): take the initial position of the particles as their best positions, marked as $P_{\mathrm{i}}(0)$; meanwhile, select the optimal as the best global position, marked as $P_{\mathrm{gt}}(0)$. Suppose $\mathrm{t}=1$;

3 ) If the optimal condition is satisfied (i.e., the adaptive value meets the required precision) or reaches the maximum iterations, execute 7), otherwise execute 4);

4) Calculate the velocity of the particle $V_{\mathrm{id}}(\mathrm{t}+1)$ and position $P_{\mathrm{id}}(\mathrm{t}+1)$ with formulas (3) and (4);

5) Compare the adaptive value of each particle with the best position $P_{\text {id }}(\mathrm{t})$ it has experienced; if better, take it as the best current position $P_{\mathrm{i}}(\mathrm{t}+1)$; traverse all the particles and compare the adaptive values with the best positions $P_{\mathrm{gt}}(\mathrm{t})$ they have experienced; if better, take it as the best current position $P_{\mathrm{gt}}(\mathrm{t}+1)$;

6) Back to 3);

7) Output the optimal result $P_{g t}(t+1)$, as well as the optimal adaptive value, the error, the computing time, etc. The optimization ends. 


\section{Simulation of Shafting Turning Errors and PSO Calculation}

As introduced above, if we need to measure the variation of the axis of rotation, it is required to know the change rule of the space position of some points. Once the position and the rotation angle are determined and the equation of the axis of rotation $L c$ is set, the changes of the space position of these points could be calculated with formulas (1) and (2). Under this way of thinking, the turning axis Lc equation could be optimized with PSO calculation.

In order to verify the correctness of PSO calculation of turning errors, imitation of turning errors is conducted here. First, set six feature points, rotating once every $6^{\circ}$. The encircled turning axis is disturbed randomly, obtaining the sequence of space position coordinate of all the feature points. After that, solve the turning axis equation with disturbance with the sequence and PSO calculation. The space coordinates of these six feature points are shown in Table 1. Suppose the turning axis passes point $\left(x_{0}, y_{0}, z_{0}\right)=(3.0000,1.0000,0.0000)$, the direction vector of the central rectilinear direction is $(m, n, p)=(-1.5000,2.6000,0.8000)$, and the amplitude of the added disturbance is the sinusoidal quantity of 0.1 and the evenly distributed random number of sinusoidal quantity 0.1 . Record every direction vector of the central rectilinear direction with disturbance on angles of $0 \sim 360$ (altogether 60 angle positions).

Table 1. Imitation of Optimization of feature point coordinates sequence

\begin{tabular}{c|c|c|c}
\hline Point number & X-coordinate & Y-coordinate & Z-coordinate \\
\hline P1 & -1.0000 & -1.0000 & -1.0000 \\
\hline P2 & 1.0000 & -1.0000 & 2.7500 \\
\hline P3 & -1.0000 & 1.0000 & 7.5000 \\
\hline P4 & 2.0000 & 0.0000 & 1.3750 \\
\hline P5 & 0.0000 & 2.0000 & -8.8750 \\
\hline P6 & 1.2000 & 1.5000 & -5.0000 \\
\hline
\end{tabular}

Conduct particle swarm optimization of the above imitation data point coordinate, the optimization parameters are $\left(x_{0}, y_{0}, z_{0}\right)$ and $(m, n, p)$. In PSO, parameters are limited and simplified. First suppose $z_{0}=0$ and $-1 \leq m, n, p \leq 1$; thus, the parameters are simplified to 5 , and the scopes of $m, n, p$ are diminished efficiently to avoid low searching efficiency or sinking into local optimal solution.

This research compiled PSO calculation software with $\mathrm{VC}++2010$ integrated development environment. The optimization result of the above turning error curve is as shown in Figure 3 and the calculation software is shown is Figure 4. With this software, calculation of the changing rules of shafting rotary axis, i.e., the shafting turning errors, through various feature points. 


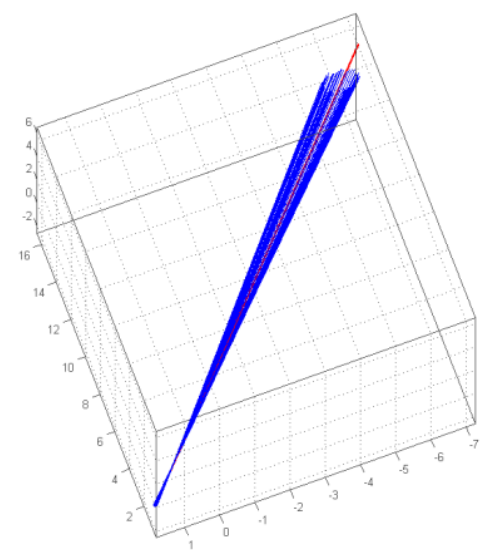

Figure 3. PSO results of shafting rotary axis

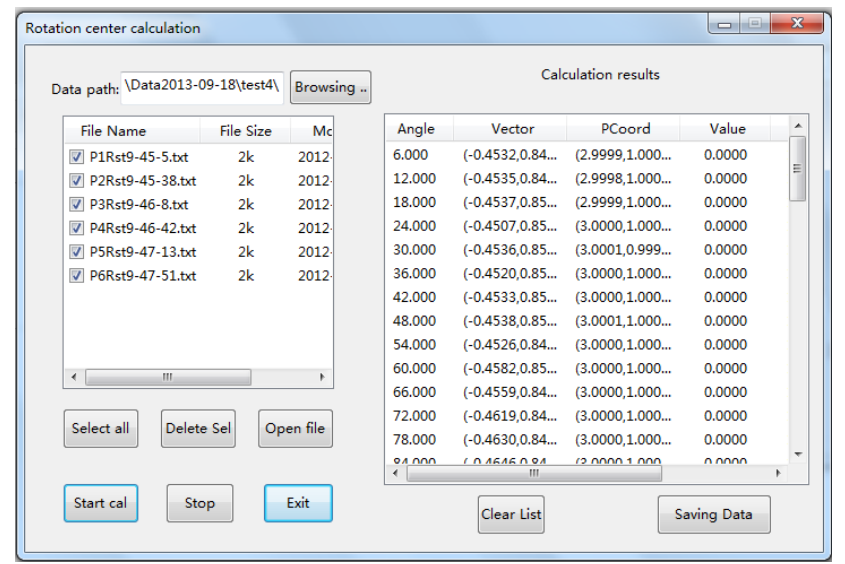

Figure 4. PSO calculation software of shaft rotation axis

\section{Rotational Error Experiment and Result Calculation}

In this paper, the experimental device is the same as reference [4]. We use atomic force microscope as the 3D sensor in measuring apparatus, made by Ambios Company of U.S. The maximum measuring ranges of this AFM are $80,80,17 \mu \mathrm{m}$ in $\mathrm{X}, \mathrm{Y}$ and $\mathrm{Z}$ direction respectively, the resolution of $X$ and $Y$ direction are $1 \mathrm{~nm}$, and the resolution of $\mathrm{Z}$ direction is $0.1 \mathrm{~nm}$. The tested precision spindle is an air-bearing rotary stage, ABRS-150MP type, made by Aerotech Inc., U.S.

Before the measurement experiment, a very important work is aligning the rotation center of the sample (i.e., precision spindle center) in the measurement region of AFM, and then move some certainly micro-structure of sample to the center of rotation. When the aligning work mentioned above is completed, the experiment of rotational error measurement can be carried out.

In this paper we only do some simple validation test. We set the AFM starts scanning in contact mode, the typical measurement range $10 * 10 \mu \mathrm{m}$, the scanning frequency is $1.5 \mathrm{~Hz}, 300 * 300$ sampling points, and measure 12 surface topography images along the circumference. Two of them are shown in Figure 5, where in Figure 5 a) d) are the 
surface topography images when the spindle at the initial $0^{\circ}, 90^{\circ}, 180^{\circ}, 270^{\circ}$ position (No. 1, 4, 7,10 image in the total of 12 images).

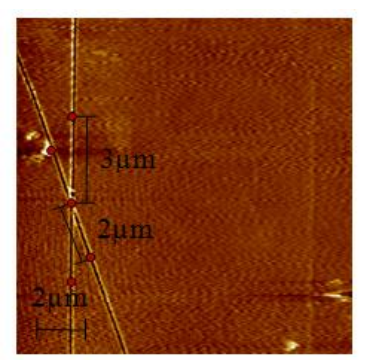

a) First image at 0 degree

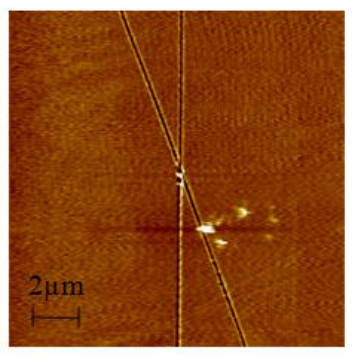

c) Image at 180 degree

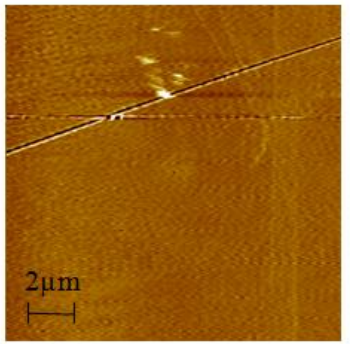

b) Image at 90 degrees

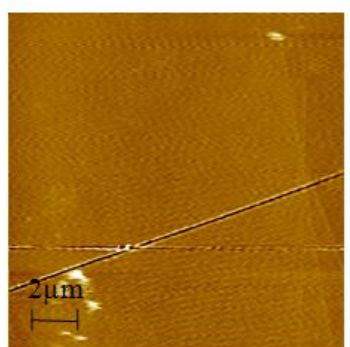

d) Image at 270 degrees

\section{Figure 5. AFM topography images of the sample}

From these AFM images, we first extract some feature points (at least five points, as shown in Figure 5 (a)) obtain three-dimensional coordinates of these points respectively, then use PSO calculation software we can calculate the curve of the measured rotation axis, as shown in Figure 6. Further separation error can be obtained radial and angular runout error curve of this shaft.

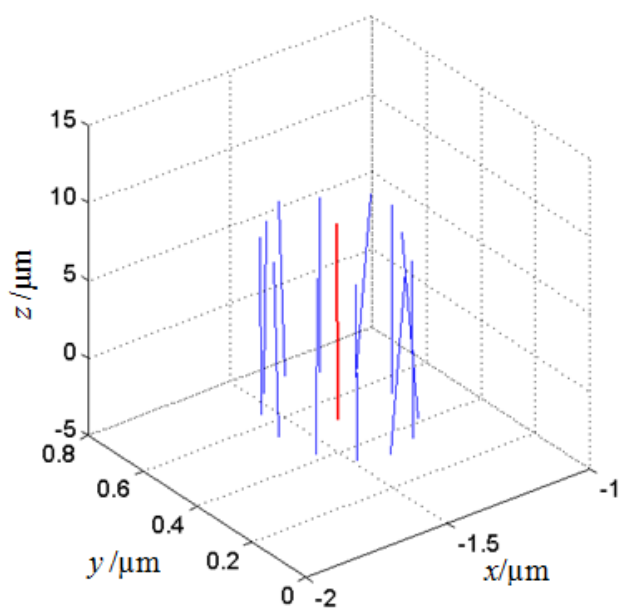

Figure 6. Experimental result of the rotational error analysis 


\section{Conclusion}

Based on the principle of turning error measurement through turning flash catch and analysis of the feature sample end shape with 3-D high accuracy sensor, the turning error solving is transformed into multi-parameter optimization of axis position of rotation with 3-D shape data. This essay takes particle swarm optimization as the way to solve this problem and introduces the procedure of the optimization. Imitation test of the rotation rules of axial system was conducted, PSO calculation was used to solve and verify the optimization and PSO analysis software for turning error optimization was also prepared. Finally, a simple example of rotational error measurement experiment by AFM is analyzed and obtain the rotational error results of measurement experiment.

\section{Acknowledgements}

This work was supported by the National Natural Science Foundation of China (Grant No. 51005061). The authors are pleased to acknowledge the contributions of Dr. Yan Yongda, Sun Tao,Geng Yanquan and Dong Shen at the Precision Engineering Research Institute of Harbin Institute of Technology. This work was also performed under the algorithm support of the School of Basic Science of Harbin University.

\section{References}

[1] S. Y. Li and Y. F. Dai, "On-line measurement and error isolation techniques of precision and ultra-precision machining", National University of Defense Technology, Changsha, vol. 3, (2007), pp. 190-202.

[2] G. Robert, E. Marsh and R. Vallance, "Precision Engineering, vol. 29, (2005), pp. 113-123.

[3] ANSI B89.3.4, Axes of Rotation: Methods for Specifying and Testing Standard, ANSI/ASME, (2004).

[4] X. S. Zhao, T. Sun, Y. D. Yan, Z. J. Hu and S. Dong, "The 10th International Symposium of Measurement Technology and Intelligent Instruments, Daejeon. Korea, (2011) June 29-July 2.

[5] X. S. Zhao, T. Sun, Y. D. Yan, Z. J. Hu, Y. D. Wu and S. Dong, "Morphology registration analysis-based method for detecting precision of precise main shaft rotation", CN, Patent 102252617B, (2013) January 16.

[6] J. Kennedy and R. C. Eberhart, "Particle swarm optimization", Proceedings of IEEE International Conference on Neural Networks", New York, U. S. A., (1995) November 27-December 1, pp. 1942-1948.

[7] J. Kennedy, "The Particle Swarm: Social Adaptation of Knowledge", Proc. IEEE, International Conference on Evolutionary Computation, Washington, DC, USA, (1997) April 13-16, pp. 303 -308.

[8] J. C. Zheng, J. Jie and Z. H. Cui, "Particle swam optimization Algorithm", Science Publishing Company of Beijing, Beijing, (2004).

[9] M. Clerc and J. Kennedy, IEEE, Transactions on Evolutionary Computation, vol. 6, (2002), pp. 58-73.

[10] X. Yu, M. F. Huang and P. Xia, "Computer System and Application”, vol. 12, (2009), pp. 201-203.

[11] Y. M. Liu, C. L. Sui and Q. Z. Zhang, "System engineering theory and practice", vol. 31, (2011), pp. 512-521.

[12] S. Gao, J.Y. Yang and X. J. Wu, "Computer Applications and Software”, vol. 22, (2005), pp. 103-104. 